\title{
Pilot Study
}

\section{Feasibility Study of Rapid Opioid Rotation and Titration}

\author{
Marina Korkmazsky, MS1', Javid Ghandehari, MD 2 ,Angela Sanchez, BS1, \\ Huong-Mo Lin, DrSc ${ }^{1}$, and Marco Pappagallo, MD ${ }^{1}$
}

From: ${ }^{1}$ Mount Sinai Hospital and School of Medicine, Department of Anesthesiology, New York, NY; ${ }^{2}$ Maimonides Medical Center, Brooklyn, NY.

Ms. Korkmazsky is Clinical Research Coordinator with The Mount Sinai Medical Center, Voorhees, NJ.

Dr. Ghandehari is an Anesthesiology Resident with Maimonides Medical Center, Brooklyn NY.

Ms. Sanchez is Senior Clinical Research Coordinator with The Mount Sinai Medical Center, New York, NY.

Dr. Lin is Associate Professor with the Department of Anesthesiology, The Mount Sinai Medical Center New York, NY.

Dr. Pappagallo is a Professor with The Mount Sinai Medical Center, New York, NY.

Address correspondence: Marco Pappagallo, MD

Professor of Anesthesiology Department of Anesthesiology The Mount Sinai Medical Center New York, NY 10029 USA

Email: marco.pappagallo@mssm.edu

Disclaimer: There was no external funding in the preparation of this manuscript.

Conflict of Interest: Dr. Pappagallo has received grants from Endo Pharmaceuticals Inc., Chadds Ford, PA. $\mathrm{He}$ is a consultant for GlaxoSmithKline,

Alkermes, Pfizer, NeurogesX, Purdue Pharma, and Baeta. All other authors attest that they have no conflicts of interest to disclose.

Manuscript received: $08 / 17 / 2010$ Revised manuscript received: $11 / 16 / 2010$

Accepted for publication: 12/17/2010

Free full manuscript: www.painphysicianjournal.com
Background: Opioid guidelines recommend opioid rotation and switching for patients who do not achieve adequate pain relief or who experience intolerable adverse events (AEs) with their current opioid. However, specific recommendations and protocols for opioid rotation are lacking, making the practice time consuming and difficult for primary care physicians to accomplish independently or coordinate with a pain specialist.

Objectives: To assess the safety and feasibility of using 24-hour intravenous patientcontrolled analgesia (IV-PCA) to achieve rapid opioid rotation and titration (RORT).

Study design: Open-label pilot study.

Setting: Hospital research center.

Methods: At admission, patients (aged $\geq 18$ years) with treatment-refractory chronic pain who were taking morphine or oxycodone for $\geq 3$ months and had pain scores $\geq 4$ on a 10 point scale, underwent opioid rotation to oral oxymorphone extended release (ER). They also received IV-PCA oxymorphone for 24 hours as needed. At discharge, the participants were taking oral oxymorphone ER with oxymorphone immediate release (IR) as needed based on their total 24-hour oral plus IV-PCA oxymorphone use. During a 2-week follow-up, their oxymorphone usage was titrated as needed. Main outcome measures were AEs, Patient Global Impression of Change (PGIC), Brief Pain Inventory $(0=$ no pain/interference, $10=$ worst pain/ complete interference), treatment satisfaction, and change in oxymorphone dose.

Results: Twelve patients enrolled and completed the 24-hour IV-PCA; 10 completed the 2week follow-up post-24-hour IV-PCA. PGIC status improved by 12 hours (odds ratio [OR], $0.19,95 \% \mathrm{Cl}, 0.08-0.44 ; P<0.001)$, and both PGIC status and activity scores improved by 24 hours (OR, $0.23,95 \% \mathrm{Cl}, 0.09-0.55 ; P=0.001 ; \mathrm{OR}, 0.49,95 \% \mathrm{Cl}, 0.25-0.96 ; P=$ 0.04 , respectively) and 2 weeks $(\mathrm{OR}, 0.14,95 \% \mathrm{Cl}, 0.04-0.46 ; P=0.001 ; \mathrm{OR}, 0.21,95 \%$ $\mathrm{Cl}, 0.06-0.72 ; P=0.01$ ) versus 6 hours. During the 24-hour IV-PCA time period, 6 of 10 patients accomplished $\geq 50 \%$ of their overall dose titration. At 2 weeks, 8 of 10 participants were Greatly Satisfied or Somewhat Satisfied with the overall RORT procedure. RORT was well tolerated, with no serious AEs.

Limitations: This was a pilot open-label study in a small number of participants. A larger randomized study with long-term follow-up and comparison to traditional protocols is necessary.

Conclusions: Preliminary data suggest that RORT can be performed safely and effectively by incorporating IV-PCA during the first 24 hours. Further investigations are needed to determine whether RORT can become an ambulatory treatment intervention in pain practice.

Clinicaltrials.gov registration: NCT00580294

Key words: chronic pain, opioid rotation, opioid switching, opioid substitution, opioid conversion, oxymorphone, oxycodone, morphine

Pain Physician 2011; 14:71-82 


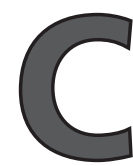
hronic opioid therapy is a potential therapeutic option in selected patients with moderate to severe chronic non-cancer pain for whom alternative therapies are less favorable (1). Even today, despite new treatments, a number of patients with chronic non-cancer pain might achieve unsatisfactory pain relief from a combination of nonopioid agents and might require the addition of opioid analgesics to their regimen (1). Nevertheless, there is controversy regarding the use of opioids for chronic non-cancer pain that is linked to questions about effectiveness and concerns about adverse events (AEs) (2). Opioid therapy is commonly associated with AEs of nausea, vomiting, constipation, sedation, and mental cloudiness; over time, tolerance develops to most of the AEs. With chronic use, endocrine dysfunction and analgesic tolerance/hyperalgesia might also occur (3-5). Opioid abuse and poisoning can also be considered AEs; both have increased over the last decade, coincident with increased availability and use of opioid analgesics (6). Addiction presents a risk to a minority of patients (4), and this risk can be mitigated with attentive screening and adherence monitoring $(1,7,8)$. When used appropriately, opioids can provide pain control with less organ toxicity than other analgesics, such as nonsteroidal antiinflammatory drugs and acetaminophen $(9,10)$.

Successful opioid treatment depends on achieving a favorable balance between analgesia and AEs. Patients vary in their response to different opioids (11), and opioid switching or rotation may be used because a poor analgesic response or intolerable AEs occur initially, or when a patient experiences reduced efficacy after an initially successful treatment (12-14). An estimated $15 \%$ to $30 \%$ of patients first prescribed morphine will need to switch to an alternate opioid $(12,15)$. A chart review by Quang-Cantagrel (13) determined that 63\% of patients (54/86) prescribed long-acting opioids required a switch because of intolerable AEs or inadequate analgesia (13). Patients might need multiple rotations to achieve effective analgesia with tolerable AEs(15). Rotation to a new opioid can improve analgesia, reduce AEs, and is clinically considered a useful treatment strategy $(12,15,16)$.

A national survey of primary care physicians, pain physicians, chiropractors, and acupuncturists determined that primary care physicians treat the majority of chronic pain patients (52\%) but are least likely to be confident in their ability to manage chronic pain (17). Many primary care physicians believe that their training does not prepare them adequately to manage important aspects of chronic pain care, including opioid switching and rotation
$(18,19)$. Guidelines on opioid rotation and switching are limited $(20,21)$, and primary care physicians often must consult with a pain specialist. Switching might require weeks, and rotation might require months (12). Inadequate access to pain specialists and difficulty achieving continuity of care with the same provider are common issues that can make opioid rotation and switching difficult and time consuming for the primary care physician, whether undertaking it independently or coordinating it with a pain specialist (18).

A short-term opioid rotation protocol (ideally $<8$ hours) that can be performed in the outpatient setting would enable primary care physicians to defer the interventional aspect and expert management of opioid switching and rotation to a pain specialist. This pilot study evaluated whether opioid switching or rotation could be accomplished safely and successfully using < 24 hours of intravenous (IV) patient-controlled analgesia (PCA) followed by a 2-week oral dose adjustment phase (rapid opioid rotation and titration [RORT]); this represents a short-term aggressive pharmacologic intervention to safely reduce time required. We believe that there would be physician interest in such an intervention, even if it requires changes to current practice.

The safety and feasibility data are intended to provide a foundation for future investigations of RORT outpatient procedures.

\section{Methods}

\section{Study Design}

This open-label pilot study conducted at Mount Sinai School of Medicine, Department of Anesthesiology, Clinical Research Center (CRC), New York, NY, enrolled patients from August 10, 2007 to August 9, 2008, and was approved by the Mount Sinai Internal Review Board (IRB; GCO \#07-0464 AN). All participants provided written informed consent. The study comprised 2 periods. During period 1 , participants were admitted to the CRC and switched to oxymorphone extended release (ER) via both oral and intravenous patient-controlled analgesia (IV-PCA) oxymorphone (see Treatment Regimen section). After 24 hours, participants were discharged with oral oxymorphone ER and oxymorphone immediate release (IR) as needed and commenced period 2, which was the final 2 weeks of the RORT procedure. The 24-hour inpatient admission time frame was a safety requirement of the IRB. The 2 main co-primary endpoints were overall safety and the change in the Patient Global Impression of Change (PGIC). 


\section{Patient Population}

Inclusion criteria were age $\geq 18$ years and a diagnosis of moderate to severe ( $\geq 4$ on a $0-10$ pain intensity scale [ $0=$ no pain, $10=$ worst pain]) chronic ( $\geq 3$ months) non-cancer pain. Participants had to be taking morphine (total daily dose $\geq 60 \mathrm{mg}$ ) or oxycodone (total daily dose $\geq 30 \mathrm{mg}$ ). Previous attempts to improve pain control by increasing the opioid dose were to have been followed by worsening of opioid-induced AEs. Participants could not have a history of alcohol or drug dependence or abuse, nor display drug-seeking behaviors or aberrant patterns of drug use.

Exclusion criteria included pregnancy or breast-feeding; diagnosis of sleep apnea or clinical features suggestive of sleep apnea; abnormal electrocardiogram; clinically significant cardiac, renal, hepatic, or lung disease; abnormal blood work; hypersensitivity to oxymorphone ER; major depression, anxiety, or insomnia resulting in daily benzodiazepine or hypnotic drug use; score $<26$ points on the Mini Mental State Examination; bedridden state; or inadequate ability to speak English.

\section{Treatment Regimen}

No additional morphine or oxycodone was dispensed at the CRC, and no changes to nonopioid medication were made during the study. Details of the RORT treatment regimen are provided in Fig. 1. During period 1, patients were switched to oxymorphone ER, with oral oxymorphone ER as the basal opioid during the first 24 hours and supplemental IV-PCA oxymorphone as needed. The calculated basal dose of oxymorphone ER was $50 \%$ of the oral dose that was equianalgesic to the participant's previous opioid dosage, determined using the conversion table in the oxymorphone ER prescribing information (22). The basal dose was divided into 2 doses, with one given at time 0 and the other approximately 12 hours later. The IV-PCA oxymorphone dose was $10 \%$ of the calculated equianalgesic dose based on the hourly oral morphine equivalent dose of the participant's previous opioid. The IV-PCA bolus dose of oxymorphone was permitted every 6 minutes as needed to a maximum of 10 doses per hour; throughout period 1 , the study physician titrated the dose up to increase ef-

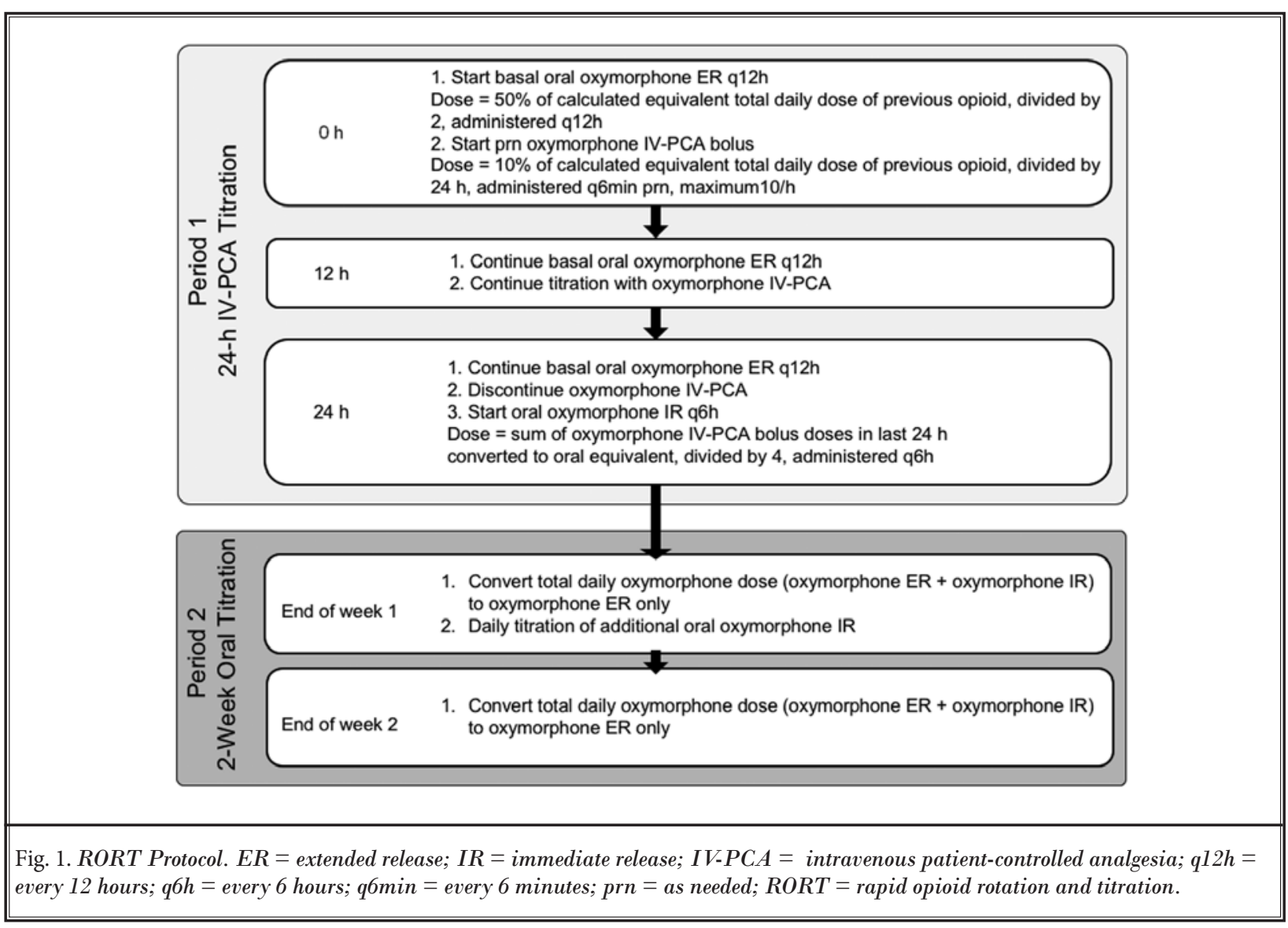


fectiveness, or down based on AEs. Participants were encouraged to sit or walk and to use the IV-PCA to control all pain episodes. If any unmanageable AEs occurred, IVPCA was discontinued. Upon discharge from the CRC following period 1, participants received a combination of oral oxymorphone ER and oxymorphone IR, which was calculated based on the cumulative oxymorphone dose administered over the previous 24 hours ( $1 \mathrm{mg}$ IV $=10$ $\mathrm{mg}$ oral) (22). The accuracy of the conversion was limited to within $5 \mathrm{mg}$ because this is the smallest available dose of oxymorphone IR. During period 2, participants underwent a 2-week oral titration. The study coordinator called daily to assess pain relief and AEs. The oxymorphone ER and IR dosages were adjusted up or down as needed to maintain pain control ( $\geq 2$-point decrease on the Brief Pain Inventory [BPI] average daily pain scale vs baseline) while minimizing AEs. At the end of period 2, participants returned to the CRC for the final study visit.

\section{Safety Assessments}

Safety was the first of 2 coprimary endpoints. AEs, pulse oximetry, and vital signs were evaluated every hour until the sixth hour of IV RORT, then every 2 hours until the 12th hour, and then every 4 hours until the 24th hour. Study personnel queried participants about the occurrence of AEs during both RORT periods. AEs were categorized by intensity (mild, moderate, or severe) and relationship to the study medication. Serious AEs (SAEs) were defined as those that required hospitalization for $>24$ hours, were life threatening, or resulted in significant disability.

\section{Effectiveness Assessments}

Treatment effectiveness, as assessed by change in PGIC score, was the second coprimary endpoint. For this endpoint, participants answered 2 questions regarding change in overall status and overall activity from baseline using a 7-point scale (1 = Very Much Improved, 2 = Much Improved, 3 = Minimally Improved, $4=$ No Change, 5 = Minimally Worse, $6=$ Much Worse, $7=$ Very Much Worse). PGIC scores at the sixth hour were compared with scores at the 12th and 24th hour of period 1 and with scores at the end of period 2 .

Secondary endpoints included the Numeric Rating Scale (NRS; $0=$ no pain, $10=$ worst imaginable pain), average daily pain, and pain interference with daily life (questions $5-11 ; 0=$ does not interfere, $10=$ completely interferes) from the BPI. During period 1, pain was assessed using the NRS hourly until the sixth hour, every 2 hours until the 12th hour, and then every 4 hours through the 24th hour, with appropriate dose adjustment. Participants also recorded average daily pain and pain interference with daily life (BPI) at baseline/enrollment, at the 24th hour of period 1, and daily during period 2 .

At the final visit, participants responded to a 15item questionnaire related to satisfaction with various aspects of treatment ( 1 = Much Better; 2 = Somewhat Better; 3 = About the Same; 4 = Somewhat Worse; 5 = Much Worse) and overall satisfaction with the RORT (1 = Very Satisfied; 2 = Satisfied; 3 = Neutral; 4 = Dissatisfied; 5 = Very Dissatisfied).

\section{Statistical Analysis}

The Cochran-Mantel-Haenszel test was used to evaluate the overall association between PGIC-one of 2 main co-primary endpoints-and time, while controlling for individual effects. The generalized estimating equations method was used to fit a cumulative logit model to the ordinal data with the PGIC as the response and time as a covariate. The odds ratio (OR) indicates the relative differences between the time points. Movement toward a lower score shows improvement.

For the secondary effectiveness endpoints, BPI and NRS, a piecewise random coefficients model was used to model the mean pain intensity over the multiple assessments. For each period before and after the infusion, the individual response of each participant was modeled as a line with intercept and slope, and an average trend line was modeled for the entire group. The slopes were contrasted to compare the change in response over time before and after the infusion and to assess any overall shift in pain score due to oxymorphone. Based on the fitted linear lines for the 2 periods, estimates were derived for the differences between values at baseline, 24th hour of period 1, and 2 weeks post-period 1. In addition, a responder analysis was performed. BPI responders for average daily pain were defined as participants with a pain reduction of $\geq 30 \%$ or 2 points (23) since baseline; BPI pain interference responders were defined as participants with a pain reduction of $\geq 1$ point on the mean of questions 5 to 11 since baseline (24).

Wilcoxon signed rank sum tests were used to evaluate the quantities of opioids administered in units of morphine-equivalent dose for periods 1 and 2 . In addition, 2 types of signed rank tests were used to determine the change in oxymorphone dose during period 1 versus period 2. Test 1 determined whether the dose increase in period 2 was greater than the dose increase in 
period 1. Test 2 determined whether the dose increase during period 1 was $\geq 50 \%$ of the total dose increase by the end of week 2 . The oxymorphone dose at the end of period 1 was defined as the equivalent oral dose of oxymorphone at the time of discharge from the CRC, calculated as the basal oral dose of oxymorphone ER plus the calculated oral oxymorphone equivalent of the IV oxymorphone dose administered by PCA during period 1 (22).

If final-visit PGIC scores were missing, all other PGIC scores were excluded. BPI average daily pain and pain interference scores were analyzed using the random coefficient model, which used all available data from all participants starting from one day post-period 1. The responder analysis and satisfaction questionnaire used a data-counting frequency for the data available at ei- ther one day post-period 1 or at the end of period 2. All evaluations were performed using SAS/STAT Version 9.1 (SAS Institute Inc., Cary, NC).

\section{Results}

\section{Participant Demographics and Disposition}

Twelve patients were enrolled; at baseline, 8 were taking morphine and 4 were taking oxycodone. All 12 participants had tried alternate pain medications (opioid and nonopioid) in the past and/or were taking nonopioid pain medication complementary to their opioid therapy at enrollment (Table 1). Participant flow is shown in Fig. 2. All 12 participants completed period 1 and 10 completed period 2 . The 2 participants who dropped out had a diagnosis of failed back syndrome.

Table 1. Opioid and nonopioid medication use and opioid-induced AEs observed at baseline or before study

\begin{tabular}{|c|c|c|c|}
\hline Patient & $\begin{array}{l}\text { Baseline } \\
\text { Opioid* }\end{array}$ & $\begin{array}{l}\text { Reasons for Unsatisfactory } \\
\text { Pain Relief at Baseline } \\
\end{array}$ & $\begin{array}{l}\text { Pain Medications Reported at } \\
\text { Baseline or Before Study } \dagger\end{array}$ \\
\hline 1 & Morphine & $\begin{array}{l}\text { Decreased opioid efficacy } \ddagger \\
\text { Unmanageable opioid-induced AEs } \\
\text { (drowsiness) }\end{array}$ & $\begin{array}{l}\text { Duloxetine, epidural steroids, methylphenidate, morphine, } \\
\text { pregabalin }\end{array}$ \\
\hline 2 & Morphine & Decreased opioid efficacy $\ddagger$ & $\begin{array}{l}\text { Amitriptyline, clonazepam, duloxetine, IV lidocaine, metha- } \\
\text { done, milnacipran, morphine, pregabalin }\end{array}$ \\
\hline 3 & Morphine & $\begin{array}{l}\text { Unmanageable opioid-induced AEs (constipa- } \\
\text { tion, drowsiness) }\end{array}$ & $\begin{array}{l}\text { Duloxetine, fentanyl patch, gabapentin, lidocaine patch, } \\
\text { morphine }\end{array}$ \\
\hline 4 & Oxycodone & $\begin{array}{l}\text { Unmanageable opioid-induced AEs } \\
\text { (drowsiness) }\end{array}$ & $\begin{array}{l}\text { Bupropion, fentanyl patch, gabapentin, lidocaine patch, NSAID, } \\
\text { venlafaxine, oxycodone }\end{array}$ \\
\hline 5 & Morphine & $\begin{array}{l}\text { Decreased opioid efficacy } \ddagger \\
\text { Unmanageable opioid-induced AEs (constipa- } \\
\text { tion, depression, nausea) }\end{array}$ & $\begin{array}{l}\text { Doxepin, duloxetine, gabapentin, hydrocodone nerve block, } \\
\text { morphine, pregabalin, trazodone, oxycodone }\end{array}$ \\
\hline 6 & Oxycodone & $\begin{array}{l}\text { Unmanageable opioid-induced AEs (drowsiness, } \\
\text { nausea) }\end{array}$ & $\begin{array}{l}\text { Duloxetine, fentanyl patch, gabapentin, hydrocodone, nerve } \\
\text { block, NSAID, oxycodone, tramadol }\end{array}$ \\
\hline 7 & Morphine & $\begin{array}{l}\text { Unmanageable opioid-induced AEs (constipa- } \\
\text { tion, drowsiness, nausea) }\end{array}$ & $\begin{array}{l}\text { Fentanyl patch, hydrocodone, methadone, nortriptyline, oxyco- } \\
\text { done, sertraline, topiramate, trazodone, venlafaxine, morphine }\end{array}$ \\
\hline 8 & Oxycodone & Decreased opioid efficacy $\ddagger$ & $\begin{array}{l}\text { Cyclobenzaprine, diazepam, fentanyl, methadone, morphine, } \\
\text { nerve block, oxycodone, warfarin }\end{array}$ \\
\hline 9 & Morphine & Decreased opioid efficacy $\ddagger$ & $\begin{array}{l}\text { Duloxetine, fluoxetine, hydromorphone, methadone, NSAID, } \\
\text { paroxetine, morphine }\end{array}$ \\
\hline 10 & Morphine & $\begin{array}{l}\text { Decreased opioid efficacy } \neq \\
\text { Unmanageable opioid-induced AEs (constipa- } \\
\text { tion, edema) }\end{array}$ & $\begin{array}{l}\text { Epidural steroids, hydrocodone, hydromorphone, morphine, } \\
\text { oxycodone, spinal cord stimulator, tramadol, trazodone }\end{array}$ \\
\hline 11 & Oxycodone & $\begin{array}{l}\text { Unmanageable opioid-induced AEs (constipa- } \\
\text { tion, drowsiness) }\end{array}$ & Duloxetine, gabapentin, methadone, nerve block, oxycodone \\
\hline 12 & Morphine & $\begin{array}{l}\text { Decreased opioid efficacy } \neq \\
\text { Unmanageable opioid-induced AEs (edema) }\end{array}$ & Methadone, morphine, nortriptyline, oxcarbazepine, oxycodone \\
\hline
\end{tabular}

$\mathrm{AE}=$ adverse event; $\mathrm{IV}=$ intravenous; NSAID $=$ nonsteroidal anti-inflammatory drug.

* Baseline opioid is opioid taken by participant at enrollment.

$\dagger$ Medications reported by participant as taken for pain control at baseline or at any time in the past before baseline. Opioid medications listed here include both those present at study baseline and those taken in the past but discontinued. No changes were made in the participants' nonopioid medications that were still being taken at study baseline .

¥ Clinically interpreted as due to onset of opioid tolerance. 
Both discontinuations were unrelated to study medication or AEs.

At enrollment, participants reported the following reasons for unsatisfactory pain relief on their current opioid: unmanageable opioid-induced AEs ( $n=$ $5)$, decreased opioid efficacy $(n=3)$, or both $(n=4)$. Participant demographics and clinical characteristics are shown in Table 2.

\section{Change in Oxymorphone Dose}

Changes in oxymorphone dose are summarized in Table 3. The median difference between the mean (oral equivalent) dose of oxymorphone at baseline and the mean dose of oxymorphone at the end of RORT was significant (120 mg/d [range, 0-545], in morphine-equivalent units; $P=0.004$ ). The median difference between the mean (oral equivalent) dose of oxymorphone at the end of period 1 and the final dose of oxymorphone at the end of RORT was 67.5 (range, -90 to 592.5 ) $\mathrm{mg} / \mathrm{d}(P$ $=0.053$ ).

An estimated $58 \%$ of the total titrated change in oxymorphone dose occurred during period $1(P=0.42)$, and 6 of 10 participants arrived at $\geq 50 \%$ of their overall dose titration within period 1.
Table 2. Participant demographic and clinical characteristics at baseline

\begin{tabular}{|l|c|}
\hline Characteristic & \\
\hline Mean (SD) age, $y$ & $50.4(12.4)$ \\
\hline Mean (SD) average pain intensity ${ }^{\star}$ & $7.34(1.18)$ \\
\hline Mean (SD) pain interference $\dagger$ & $6.54(2.18)$ \\
\hline Mean (SD) duration of pain, $\mathrm{y}$ & $10.3(8.8)$ \\
\hline Women, $\mathrm{n}$ & 10 \\
\hline Chronic non-cancer pain diagnosis, $\mathrm{n}$ & \\
\hline Failed back syndrome & 4 \\
\hline $\begin{array}{l}\text { Complex regional pain syndrome/reflex sympathetic } \\
\text { dystrophy }\end{array}$ & 3 \\
\hline Severe painful osteoarthritis & 2 \\
\hline Post herpetic neuralgia & 1 \\
\hline Fibromyalgia & 1 \\
\hline Severe painful polyneuropathy & 1 \\
\hline
\end{tabular}

${ }^{*}$ Brief Pain Inventory average daily pain $(0=$ no pain, $10=$ worst imaginable pain)

${ }^{\dagger}$ Brief Pain Inventory pain interference with daily life $(0=$ does not interfere, $10=$ completely interferes)

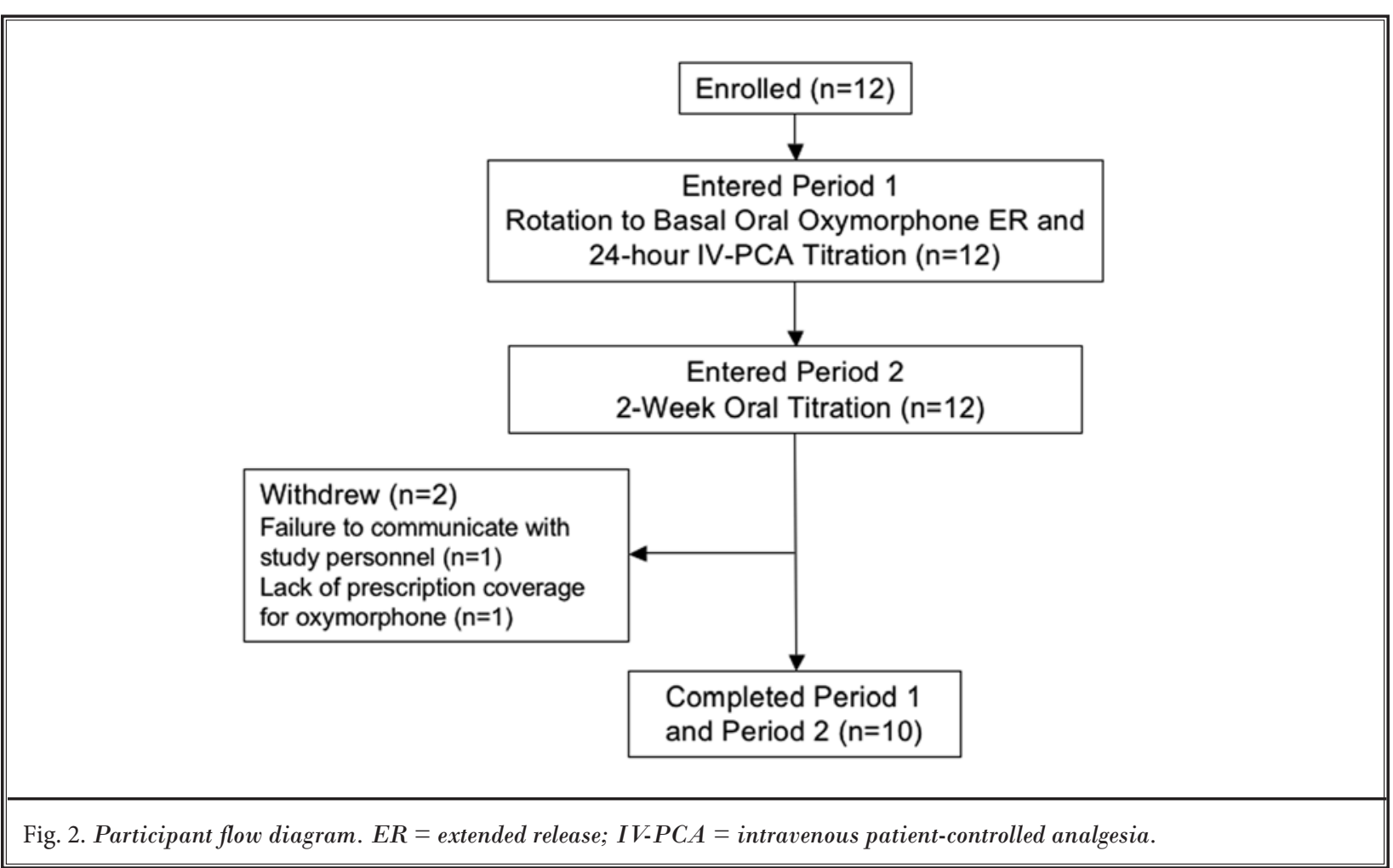


Table 3. Changes in oxymorphone dose throughout the study

\begin{tabular}{|c|c|c|}
\hline & $\begin{array}{l}\text { Changes in Oxymorphone Dose } \\
\text { (Morphine-Equivalent Units, mg/d) }\end{array}$ & $P$ Value \\
\hline \multicolumn{3}{|l|}{ Baseline dose of oral oxymorphone $\mathrm{ER}^{*}$} \\
\hline Mean (SD) & $188.4(137.4)$ & \\
\hline Range & $45-510$ & \\
\hline $\begin{array}{l}\text { Participants with a } \geq 50 \% \text { change in their overall dose titration for the entire study } \\
\text { within period } 1, \dagger \mathrm{n}(\%)\end{array}$ & $6(60)$ & \\
\hline \multicolumn{3}{|l|}{ Median (range) change in dose between RORT dosing periods } \\
\hline Change from oral baseline dose to oral equivalent dose at end of period 1 & $30(-120$ to 110$)$ & 0.386 \\
\hline Change from oral equivalent dose at end of period 1 to oral dose at end of period 2 & $67.5(-90$ to 592.5$)$ & 0.053 \\
\hline Change from oral baseline dose to oral dose at end of period 2 & $120(0-545)$ & 0.004 \\
\hline $\begin{array}{l}\text { Difference between (change from oral equivalent dose at end of period } 1 \text { to oral dose } \\
\text { period 2) and (change from oral basal dose to oral equivalent dose at end of period 1) } \ddagger\end{array}$ & $-26.3(-300$ to 495$)$ & 0.73 \\
\hline
\end{tabular}

ER = extended release; RORT = rapid opioid rotation and titration;

period 1=24-hour oral oxymorphone and IV-PCA titration; period $2=2$-week oral dose titration.

${ }^{*}$ Baseline is dose taken by participant at enrollment.

†Calculated as follows: included only participants whose change from basal dose to dose at end of period 1 was $\geq 50 \%$ of the total higher than the change from basal dose to dose period 2 .

¥stimated portion of the increase that can be attributed to the dose increase during period 1 was $58 \%(P=0.42)$. There was a trend toward greater dose increase during period 1 than during period 2 .

\section{Safety Assessments}

The RORT procedure was well tolerated; 2 of 12 participants reported new-onset AEs during period 1 (constipation, $n=2 / 12[16.7 \%]$; tiredness, $n=1 / 12$ [8.3\%]; headache, $n=1 / 12[8.3 \%]$; pruritus, $n=2 / 12$ [16.7\%]) and 3 of 10 participants reported at least one severe $A E$ (not new onset) at study exit. During period 2 , the most common severe AEs were constipation ( $n=$ $2 / 10[20 \%])$, tiredness $(n=5 / 10[50 \%])$, headaches $(n=$ $2 / 10[20 \%])$, pruritus $(n=2 / 10[20 \%])$, and nasopharyngitis $(n=1 / 10[10 \%])$. Participants reported no SAEs or new-onset severe AEs.

\section{Patient Global Impression of Change}

PGIC scores significantly correlated with time $(P=$ $0.03)$. Overall status scores improved by the 12 th hour of period 1 (OR, $0.19 ; 95 \% \mathrm{Cl}, 0.08-0.44 ; P<0.001$ ), the 24th hour of period 1 (OR, $0.23 ; 95 \% \mathrm{Cl}, 0.09-0.55 ; P$ $=0.001)$, and the end of period $2(\mathrm{OR}, 0.14 ; 95 \% \mathrm{Cl}$, $0.04-0.46 ; P=0.001$ ) compared with the sixth hour of period 1 (Fig. 3A). PGIC overall activity scores improved at the 24th hour of period 1 (OR, $0.49 ; 95 \% \mathrm{Cl}, 0.25-0.96$; $P=0.04)$ and the end of period $2(\mathrm{OR}, 0.21 ; 95 \% \mathrm{Cl}$, $0.06-0.72 ; P=0.01)$ compared with the sixth hour of period 1 (Fig. $3 \mathrm{~A}$ ). PGIC activity scores at the sixth and 12th hours of period 1 were not significantly different $(P=$ $0.44)$, although scores trended lower at hour 12 . There was no significant change in PGIC scores from the end of period 1 to the end of period 2 or from the 12th hour to the end of period 1. PGIC status and activity scores by hour during periods 1 and 2 are shown in Figs. 3B and $C$, respectively. Of note, for the PGIC status score, only one participant reported being Minimally Worse, Much Worse, or Very Much Worse at the sixth and 12th hours of period 1 (Fig. 3B); however, this participant reported being Much Improved at 2 weeks. No participant reported being worse for the activity measure.

\section{Satisfaction Questionnaire}

Treatment satisfaction at the final visit was relatively high, with 7 of 10 participants indicating that oxymorphone ER was "better" or "much better" than their previous pain medication. Furthermore, 9 of 10 participants were willing to continue using oxymorphone ER. Ratings for the overall RORT procedure were high (Greatly Satisfied, $n=6$; Somewhat Satisfied, $n=$ 2; Somewhat Dissatisfied, $\mathrm{n}=1$; Greatly Dissatisfied, $\mathrm{n}$ $=1$ ).

\section{Brief Pain Inventory}

For the BPI average daily pain and pain interference questions, the pre- and post-period 1 slopes were slightly negative but not significantly different from each other (average daily pain slope difference: $-0.03[95 \% \mathrm{Cl},-0.14$ to 0.08 ], $P=0.59$; pain interference slope difference: 0.04 [95\% Cl, -0.11 to 0.19$], P=$ 


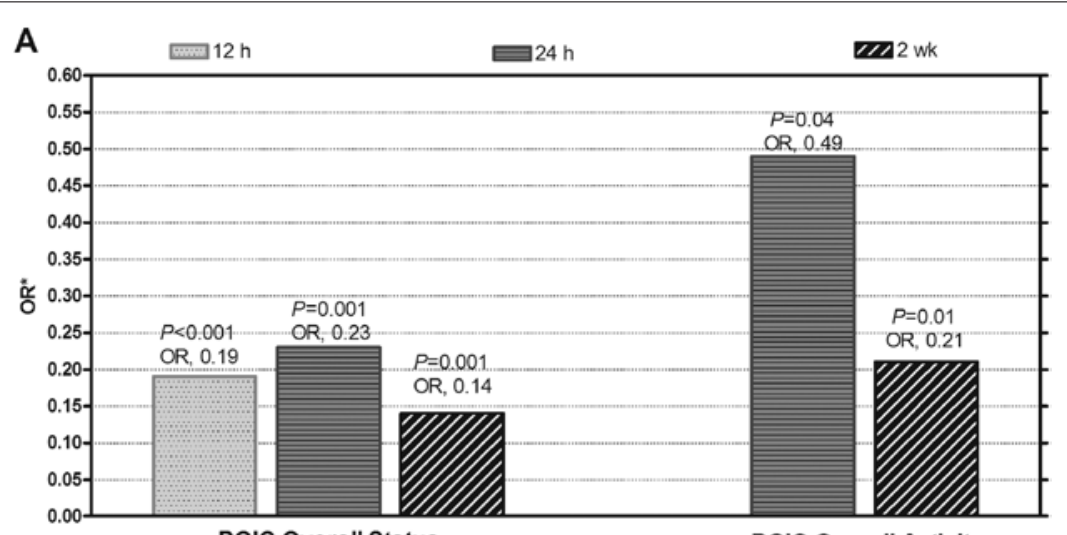

PGIC Overall Status PGIC Overall Activity
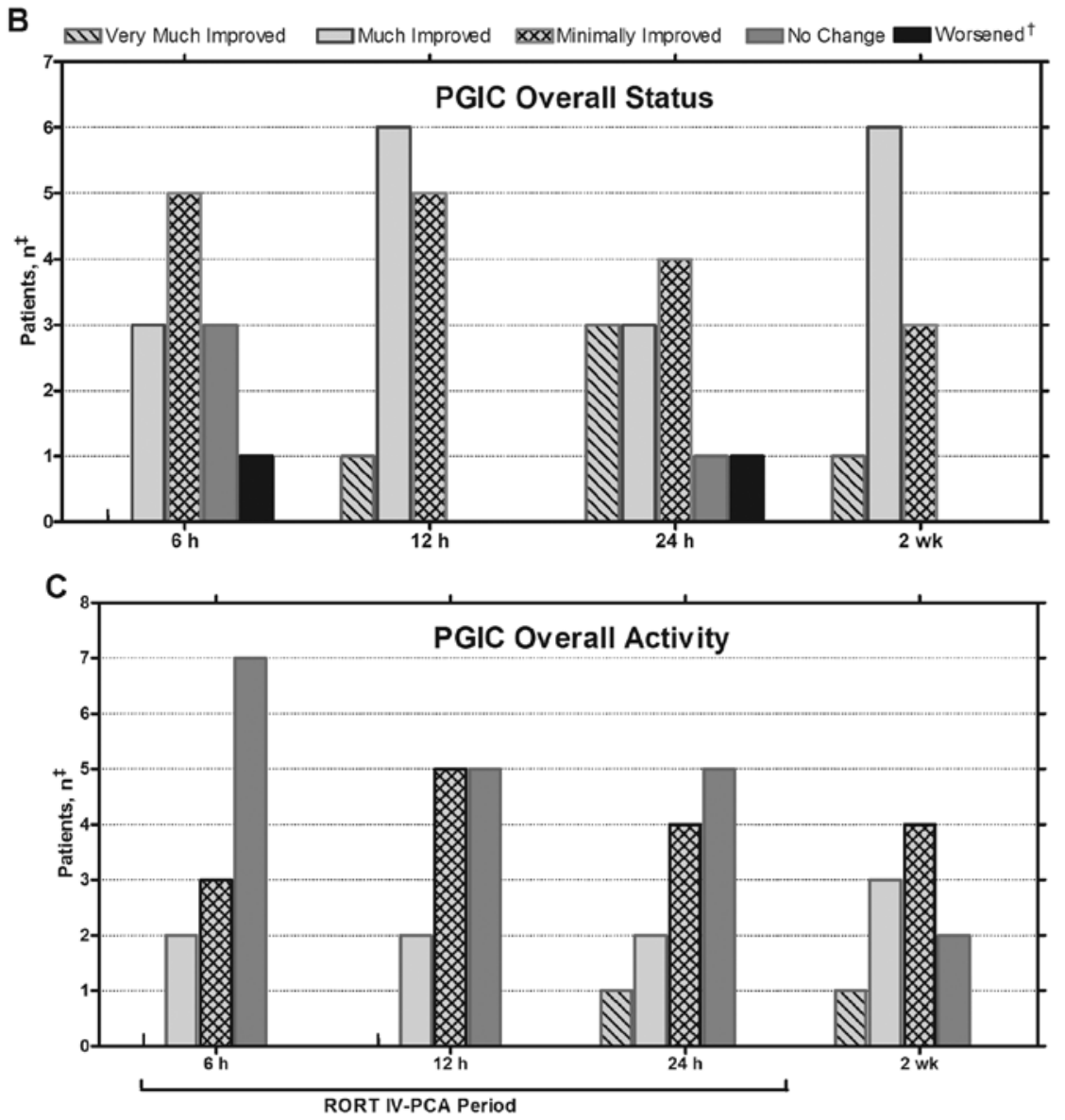

Fig. 3. Patient Global Impression of Change (PGIC) scores. A, Odds ratio (OR) for each time point vs 6 hours. B, PGIC overall status scores by hour and at 2 weeks. C, PGIC overall activity scores by hour and at 2 weeks. IV-PCA = intravenous patientcontrolled analgesia; $R O R T=$ rapid opioid rotation and titration. ${ }^{*} O R$ in comparison with the PGIC score at 6 hours. $\dagger$ Includes Minimally Worse, Much Worse, and Very Much Worse. The subject who reported worsening at 6 and 24 hours became Much Improved at 2 weeks.

$\$ 2$ participants completed the 24-hour IV-PCA titration period and then withdrew during the 2-week oral dose titration period and therefore do not have 2-week data. 
0.59). The slopes were also not significantly different from 0 , indicating that the pain had stabilized in each period. Overall, the mean $(95 \% \mathrm{Cl})$ change in average daily pain was significantly different pre- and post- period $1(-1.51[-2.79$ to -0.23$], P=0.02)$, pre-period 1 compared with one day post-period $1(-1.58$ [-2.75 to -0.42 ], $P=0.01)$, and pre-period 1 compared with the end of period $2(-2.12$ [-3.32 to -0.92$], P=0.001)$ (Fig. $4 A)$. At one day post-period 1, 7 of 12 participants were classified as responders, and at the end of period 2, 7 of 10 participants were classified as responders. Although the results were less robust for pain interference, the mean change in pain interference declined at all time points after initiation of RORT (Fig. 4B). In total, 8 of 12 participants were responders at one day post-period 1 and 7 of 10 participants were responders at the end of period 2 .

\section{Discussion}

The current exploratory pilot study was designed to evaluate whether RORT could be used to quickly convert patients from one opioid to another. Our results indicate that this method shows promise, with good effectiveness, participant satisfaction, and tolerability in participants with unsatisfactory pain relief on current opioid medication due to maximum titration or opioidinduced AEs. Only 2 participants (17\%) reported newonset AEs during the 24-hour period of oral and IV-PCA oxymorphone, and no serious AEs were reported during the study. The co-primary endpoint, PGIC scores, significantly improved by the 12th (overall status) or 24th hour (overall activity). Secondary endpoints were generally consistent, and satisfaction with the procedure and oxymorphone ER was high.

In clinical trials wherein participants with chronic non-cancer pain switched to oxymorphone ER from their previous opioid over a relatively long time period ( $\leq 4$ weeks), oxymorphone ER showed good efficacy and tolerability $(25,26)$. In the current study using a rapid rotation method, oxymorphone ER was well tolerated and effective, and participants reported a high level of satisfaction with the medication. The final regimen included both oxymorphone ER and a short-acting opioid, as needed by the participant for adequate pain control. This combination of long- and short-acting opioids is appropriate in the circumstances where breakthrough pain occurs. Direct comparison of titration of oxymorphone ER using the RORT procedure to a traditional switching and rotation protocol is an important next step.

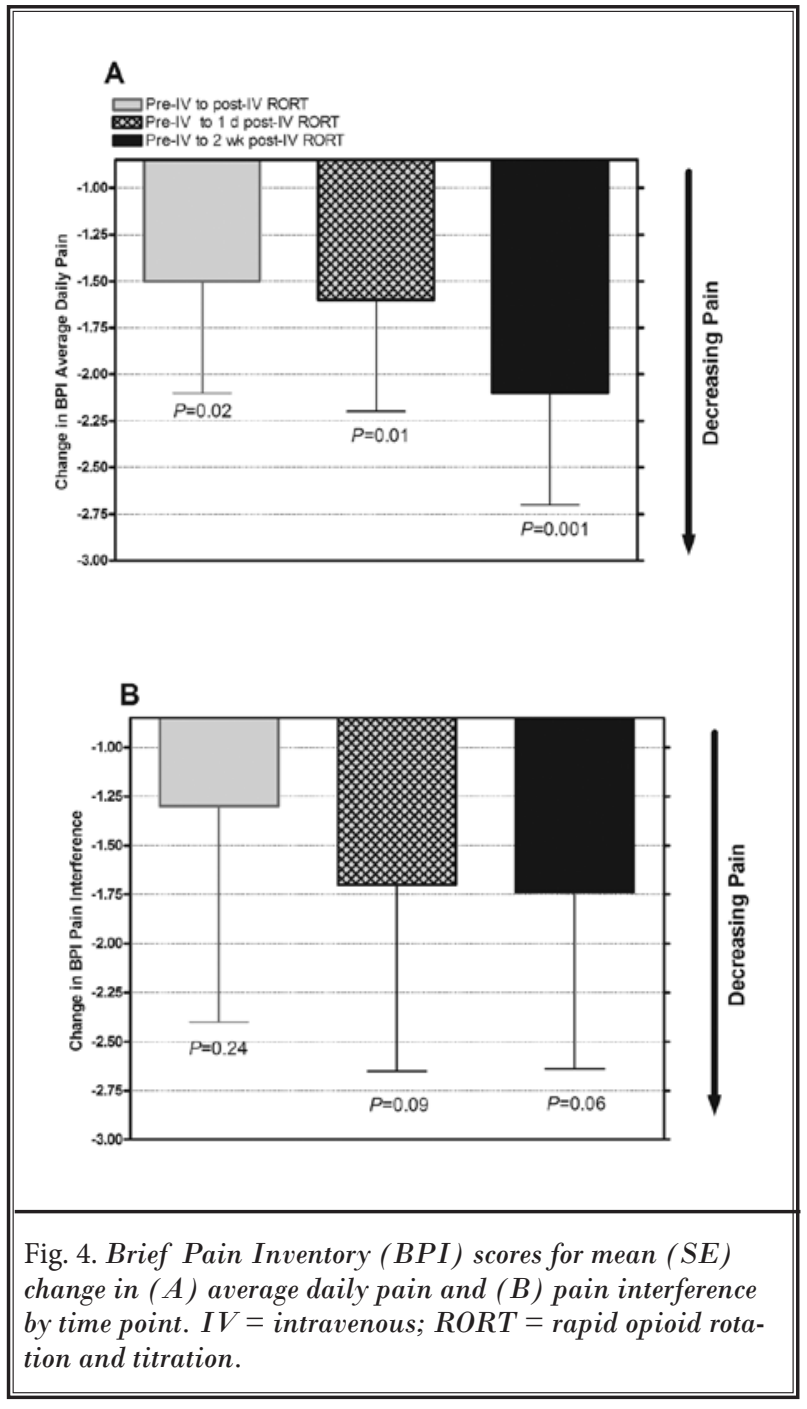

This pilot study has several limitations. First, although no participant experienced SAEs, this was a small feasibility study and larger ones are needed to affirm safety, particularly given that the 12 participants in this study had 6 different chronic non-cancer pain diagnoses. Future studies evaluating RORT in several populations with homogeneous pain diagnoses would be beneficial. Second, there was no comparator group for determining effectiveness relative to other protocols. It is also possible that the study did not adequately imitate participants' average daily level of activity and pain during the 24-hour IV-PCA. Additional studies that encourage greater physical activity during the procedure are needed to investigate this possibility; long-term follow-up is also desirable. The results show only a trend 
toward the majority of the titration occurring during period 1 (24-hour oral oxymorphone and IV PCA) versus the subsequent 2-week oral adjustment phase. However, it is important to note that, for safety reasons, the basal dosage of oxymorphone ER represented a $50 \%$ reduction of participants' initial calculated equianalgesic dose of oxymorphone. Also, the significant difference in median oxymorphone dose from baseline to 2 weeks postperiod 1 is consistent with undertreatment of pain on their previous opioid; at baseline, all participants reported unsatisfactory pain relief, and increases in the previous opioid were limited by maximum titration and/or unmanageable AEs. Thus, 24-hour IV-PCA might have provided a short time frame with close monitoring in which participants could be rapidly and safely switched to a more effective yet well-tolerated dose of an alternate opioid.

When undergoing opioid switching or rotation, patients are sometimes unable to achieve proper management of pain or AEs because of the burden of multiple office visits. The ultimate goal of the RORT procedure is to streamline and improve pain management by providing a short-term outpatient procedure for chronic non-cancer pain patients who require opioid rotation and titration. Therefore, future studies will need to evaluate whether RORT can be performed in an even shorter time period (i.e., $<8$ hours) that is conducive to outpatient treatment. This was not possible in the current study; the Mount Sinai IRB required a 24-hour inpatient admission to ensure participant safety.

Although guidelines on opioid therapy for chronic pain recommend that opioid rotation be considered for patients who fail to achieve adequate pain relief or who experience intolerable AEs, guidelines for opioid rotation and switching are limited in scope, focusing on the use of equianalgesic dose-conversion tables $(20,21)$. Guidelines by the American Pain Society and American Academy of Pain Medicine for the use of chronic opioid therapy in chronic non-cancer pain make no specific recommendations for performing opioid rotation because of insufficient evidence (1). There is a very limited research base on opioid rotation and no studies comparing different approaches (27).

Historically, pain specialists have used rapid rotation methods to safely and effectively treat cancer pain emergencies; in some cases, these techniques can provide pain relief within the first 24 hours $(28,29)$. Our study in patients with chronic non-cancer pain was de- signed to implement IV-PCA to complete a RORT from other opioids to oxymorphone ER. A procedure such as RORT could begin to address concerns with current practice by enabling pain specialists to assume responsibility for the interventional aspect and expert management of opioid switching without need for protracted patient care. However, in its current form and within the framework of current practice patterns, important obstacles remain, such as reimbursement issues and the resource intensiveness of intravenous treatment. Although the RORT model offers the opportunity to safely and effectively perform opioid rotation and titration in a shorter time period, the time requirement is still an impediment to implementation. Future studies in a larger patient population should assess whether a shorter RORT protocol (< 8-hour IVPCA) can maintain the satisfaction and low rate of AEs observed here.

\section{Conclusions}

This pilot study of RORT in 12 participants achieved promising results. The transition from the previous oral opioid to oral oxymorphone ER was well controlled during the first 24 hours, and benefits were relatively rapid.

\section{Acknowledgments:}

The authors would like to thank Endo Pharmaceuticals Inc., Chadds Ford, PA, for funding this research. In addition, the authors would like to give their gratitude to Boleslav Kosharskyy, MD; Stelian Serban, MD; David Skinner, MD; and Vinoo Thomas, MD, for their assistance with study design and management as coinvestigators on the study, and Sabera Hossain, MS, for statistical analysis. Furthermore, the authors would like to acknowledge Kristine W. Schuler, MS, and Robert Gatley, MD, of Complete Healthcare Communications, Inc. (Chadds Ford, PA, USA), who provided medical writing and editorial support, which was also funded by Endo Pharmaceuticals Inc. Independently of the sponsor, the authors were responsible for study design; management of the study; collection of data; statistical analyses; interpretation of the data; and the preparation, review, and final approval of the manuscript before submission. All coauthors contributed scientifically to the manuscript, but the first author exercised editorial control with final responsibility for content decisions and conclusions. 


\section{References}

1. Chou R, Fanciullo GJ, Fine PG, Adler JA, Ballantyne JC, Davies P, Donovan MI, Fishbain DA, Foley KM, Fudin J, Gilson AM, Kelter A, Mauskop A, O’Connor PG, Passik SD, Pasternak GW, Portenoy RK, Rich BA, Roberts RG, Todd KH, Miaskowski C. Clinical guidelines for the use of chronic opioid therapy in chronic noncancer pain. J Pain 2009; 10:113130.

2. Trescot AM, Helm S, Hansen $H, B e-$ nyamin R, Glaser SE, Adlaka R, Patel $\mathrm{S}$, Manchikanti L. Opioids in the management of chronic non-cancer pain: An update of American Society of Interventional Pain Physicians' (ASIPP) Guidelines. Pain Physician 2008; 11: S5-S62.

3. Benyamin R, Trescot AM, Datta S, Buenaventura R, Adlaka R, Sehgal N, Glaser SE, Vallejo R. Opioid complications and side effects. Pain Physician 2008; 11:S105-120.

4. Chapman CR, Lipschitz DL, Angst MS, Chou R, Denisco RC, Donaldson GW, Fine PG, Foley KM, Gallagher RM, Gilson AM, Haddox JD, Horn SD, Inturrisi CE, Jick SS, Lipman AG, Loeser JD, Noble M, Porter L, Rowbotham MC, Schoelles KM, Turk DC, Volinn E, Von Korff MR, Webster LR, Weisner CM. Opioid pharmacotherapy for chronic non-cancer pain in the United States: A research guideline for developing an evidence-base. J Pain 2010; 11:807829.

5. Zacny JP, Gutierrez S. Subjective, psychomotor, and physiological effects profile of hydrocodone/acetaminophen and oxycodone/acetaminophen combination products. Pain Med 2008; 9:433-443.

6. Manchikanti L, Fellows B, Ailinani H, Pampati V. Therapeutic use, abuse, and nonmedical use of opioids: A tenyear perspective. Pain Physician 2010; 13:401-435.

7. Manchikanti L, Cash KA, Damron KS, Manchukonda R, Pampati V, McManus CD. Controlled substance abuse and illicit drug use in chronic pain patients: An evaluation of multiple variables. Pain Physician 2006; 9:215-225.

8. Fishbain DA, Cole B, Lewis J, Rosomoff $\mathrm{HL}$, Rosomoff RS. What percentage of chronic nonmalignant pain patients exposed to chronic opioid analgesic therapy develop abuse/addiction and/ or aberrant drug-related behaviors? A structured evidence-based review. Pain Med 2008; 9:444-459.

9. Zhang W, Moskowitz RW, Nuki G, Abramson $\mathrm{S}$, Altman RD, Arden $\mathrm{N}$, Bierma-Zeinstra S, Brandt KD, Croft P, Doherty M, Dougados M, Hochberg M, Hunter DJ, Kwoh K, Lohmander LS, Tugwell P. OARSI recommendations for the management of hip and knee osteoarthritis, part II: OARSI evidence-based, expert consensus guidelines. Osteoarthritis Cartilage 2008; 16:137-162.

10. Larson AM, Polson J, Fontana RJ, Davern TJ, Lalani E, Hynan LS, Reisch JS, Schiodt FV, Ostapowicz G, Shakil AO, Lee WM. Acetaminophen-induced acute liver failure: Results of a United States multicenter, prospective study. Нepatology 2005; 42:1364-1372.

11. Galer BS, Coyle N, Pasternak GW, Portenoy RK. Individual variability in the response to different opioids: Report of five cases. Pain 1992; 49:87-91.

12. Slatkin NE. Opioid switching and rotation in primary care: Implementation and clinical utility. Curr Med Res Opin 2009; 25:2133-2150.

13. Quang-Cantagrel ND, Wallace MS, Magnuson SK. Opioid substitution to improve the effectiveness of chronic noncancer pain control: A chart review. Anesth Analg 2000; 90:933-937.

14. Grilo RM, Bertin P, Scotto di Fazano C, Coyral D, Bonnet C, Vergne P, Treves R. Opioid rotation in the treatment of joint pain. A review of 67 cases. Joint Bone Spine 2002; 69:491-494.

15. McNicol E, Horowicz-Mehler N, Fisk RA Bennett K, Gialeli-Goudas M, Chew PW, Lau J, Carr D. Management of opioid side effects in cancer-related and chronic noncancer pain: A systematic review. J Pain 2003; 4:231-256.

16. Vissers KC, Besse K, Hans G, Devulder J, Morlion B. Opioid rotation in the man agement of chronic pain: Where is the evidence? Pain Pract 2010; 10:85-93.

17. Breuer B, Cruciani R, Portenoy RK. Pain management by primary care physicians, pain physicians, chiropractors, and acupuncturists: A national survey. South Med I 2010; 103:738-747.

18. Yanni LM, Weaver MF, Johnson BA, Morgan LA, Harrington SE, Ketchum JM Management of chronic nonmalignant pain: A needs assessment in an internal medicine resident continuity clinic. J Opioid Manag 2008; 4:201-211.
19. Scott E, Borate U, Heitner S, Chaitowitz M, Tester W, Eiger G. Pain management practices by internal medicine residents--A comparison before and after educational and institutional interventions. Am J Hosp Palliat Care 2008; 25:431-439.

20. Coluzzi F, Pappagallo M. Opioid therapy for chronic noncancer pain: Practice guidelines for initiation and maintenance of therapy. Minerva Anestesiol 2005; 71:425-433.

21. Fine PG, Portenoy RK. Establishing "best practices" for opioid rotation: Conclusions of an expert panel. J Pain Symptom Manage 2009; 38:418-425.

22. OPANA® ER (oxymorphone hydrochloride extended-release tablets). Full Prescribing Information. Chadds Ford, PA: Endo Pharmaceuticals Inc; 2008.

23. Farrar JT, Young JP, Jr, LaMoreaux L, Werth JL, Poole RM. Clinical importance of changes in chronic pain intensity measured on an 11-point numerical pain rating scale. Pain 2001; 94:149158.

24. Dworkin RH, Turk DC, Farrar JT, Haythornthwaite JA, Jensen MP, Katz NP, Kerns RD, Stucki G, Allen RR, Bellamy N, Carr DB, Chandler J, Cowan P, Dionne R, Galer BS, Hertz S, Jadad AR, Kramer LD, Manning DC, Martin S, McCormick CG, McDermott MP, McGrath P, Quessy S, Rappaport BA, Robbins W, Robinson JP, Rothman M, Royal MA, Simon L, Stauffer JW, Stein W, Tollett J, Wernicke J, Witter J. Core outcome measures for chronic pain clinical trials: IMMPACT recommendations. Pain 2005; 113:919.

25. Peniston JH, Gould E. Oxymorphone extended release for the treatment of chronic low back pain: A retrospective pooled analysis of enriched-enrollment clinical trial data stratified according to age, sex, and prior opioid use. Clin Ther 2009; 31:347-359.

26. Hale ME, Ahdieh H, Ma T, Rauck R. Efficacy and safety of OPANA ER (oxymorphone extended release) for relief of moderate to severe chronic low back pain in opioid-experienced patients: A 12-week, randomized, double-blind, placebo-controlled study. J Pain 2007; 8:175-184.

27. Chou R, Ballantyne JC, Fanciullo GJ, Fine PG, Miaskowski C. Research gaps on use of opioids for chronic noncancer pain: Findings from a review of the 
evidence for an American Pain Society and American Academy of Pain Medicine clinical practice guideline. J Pain 2009; 10:147-159.
28. Mercadante S, Ferrera P, Villari P, Casuccio A. Rapid switching between transdermal fentanyl and methadone in cancer patients. J Clin Oncol 2005; 23:5229-5234.
29. Mercadante S, Villari P, Ferrera P, Casuccio A, Fulfaro F. Rapid titration with intravenous morphine for severe cancer pain and immediate oral conversion. Cancer 2002; 95:203-208. 